\section{Prevalência de depressão em bombeiros}

\section{Prevalence of depression among firefighters}

\author{
Prevalencia de depresión en bomberos
}

\begin{abstract}
Depression burder is high worldwide. Socioeconomic factors and exposure to extreme situations at work may be associated with the illness. This study focused on the prevalence of depression and associated factors among firefighters in Belo Horizonte, Minas Gerais State, Brazil. A cross-sectional study was conducted among male firefighters in Belo Horizonte $(n=711)$. The Beck Depression Inventory (BDI) was used to assess depression. Univariate and multivariate logistic regression models were used to study the association between socio-demographic characteristics, occupational stressors, health status, and depression. Prevalence of depression in the sample was 5.5\%. The likelihood of developing depression was higher among firefighters who reported post-traumatic stress symptoms $(O R=12.47 ; 95 \% C I: 5.64-27.57)$ and alcohol abuse (OR = 5.30; 95\%CI: 2.35-11.96). The results are discussed considering the interrelationships between mental disorders, the healthy worker effect, and social recognition of firefighters' work.

Firefighters; Occupational Exposure; Depression; Healthy Worker Effect
\end{abstract}

Eduardo de Paula Lima 1

Ada Ávila Assunção 1

Sandhi Maria Barreto 1

\section{Resumo}

A depressão apresenta alta carga de doença no mundo. Fatores socioeconômicos e exposição a situações extremas no trabalho podem estar associados à doença. O objetivo do trabalho é investigar a prevalência e fatores associados à depressão em bombeiros de Belo Horizonte, Minas Gerais, Brasil. Estudo transversal foi realizado em universo de bombeiros do sexo masculino em Belo Horizonte $(n=711)$. O Inventário Beck para Depressão (IBD) foi utilizado para avaliar a presença de depressão. Modelos de regressão logística (uni e multivariada) foram utilizados para estudar a associação entre características sociodemográficas, estressores ocupacionais, situação de saúde e depressão. A prevalência de depressão na amostra estudada foi $5,5 \%$. A chance de depressão foi maior entre bombeiros que relataram sintomas de estresse pós-traumático (OR = 12,47; IC95\%: 5,64-27,57) e uso abusivo de álcool (OR = 5,30; IC95\%: 2,35-11,96). Os resultados são discutidos considerando as interrelações entre transtornos mentais, o efeito do trabalhador sadio e o reconhecimento social do trabalho dos bombeiros.

Bombeiros; Exposição Ocupacional; Depressão; Efeito do Trabalhador Sadio 


\section{Introdução}

A depressão é uma doença psiquiátrica recorrente e incapacitante, caracterizada por humor deprimido e perda de prazer ou interesse em atividades cotidianas 1 . Para a confirmação do diagnóstico, indaga-se ainda sobre alterações psicomotoras, cognitivas e somáticas 2 . Investigações sobre as causas da depressão sugerem uma etiologia complexa, pois vulnerabilidades individuais (biológicas e psicológicas) são influenciadas pelo macroambiente ${ }^{3,4}$.

A carga de doença é alta em diversos países 5 , sendo que fatores socioeconômicos e características regionais influenciam o padrão de adoecimento. Na África e leste da Ásia, por exemplo, sobressaem as doenças infecciosas e os problemas perinatais. No caso da região das Américas, destacam-se os episódios de depressão unipolar cuja carga de doença é alta desde 2004. Estimase que, até 2030, a depressão estará em primeiro lugar no rol das morbidades com maior carga de doença no mundo 6 .

Quanto aos fatores socioeconômicos, vínculos precários de emprego 7,8 e exposição a demandas psicossociais negativas ou excessivas no trabalho 9,10 exercem influência sobre a depressão. A vivência de situações extremas e/ou traumáticas durante a atividade laboral pode contribuir para o adoecimento 11, sendo registradas comorbidades psiquiátricas em bombeiros, socorristas e outros profissionais de emergências 12,13.

Bombeiros desempenham tarefas diversas em contextos de emergência. Combate a incêndio, atendimento pré-hospitalar e resgate de feridos em acidentes automobilísticos são exemplos de ocorrências rotineiras atendidas em centros urbanos. Tais demandas exigem respostas rápidas para assegurar a integridade física das vítimas e dos próprios trabalhadores, implicando convívio com situação extremas como morte eminente ou atendimento às zonas onde houve massacres e acidentes fatais 14 .

A exposição ocupacional a eventos de tal natureza está associada ao estresse pós-traumático, cuja prevalência pode atingir $46 \% 15$. A ocorrência de estresse pos-traumático aumenta a probabilidade de depressão em sujeitos expostos às situações mencionadas 16 .

A investigação sobre depressão em bombeiros ainda é incipiente no Brasil. Estudo exploratório em Juiz de Fora, Minas Gerais, evidenciou sintomas compatíveis com depressão em $10 \%$ dos bombeiros $(8,3 \%$ do sexo masculino; $26,9 \%$ do sexo feminino) ${ }^{17}$. A análise qualitativa dos dados (entrevistas) identificou queixas relativas à organização do trabalho. Destacaramse as seguintes: escassez ou inadequação de equipamentos, estrutura física precária, planejamento institucional insuficiente e sobrecarga de tarefas. Tais fatores estariam relacionados a problemas de saúde mental; entretanto, limitações metodológicas restringiram avanço nas hipóteses 17 .

Em Belo Horizonte, capital do Estado de Minas Gerais, o Corpo de Bombeiros conta atualmente com três batalhões e 11 subunidades. Os profissionais são admitidos por concurso público, após serem submetidos a exames de saúde e provas de força e resistência física. Ao iniciar a carreira, tais avaliações são repetidas anualmente. O vínculo empregatício é formal e as normas internas da instituição garantem aos trabalhadores estabilidade, possibilidade de ascensão na carreira e acesso a serviços de saúde. A hierarquia segue o padrão das instituições de caráter militar.

O objetivo do presente artigo é: (1) estimar a prevalência de depressão; e (2) conhecer os fatores associados à doença em bombeiros de Belo Horizonte.

\section{Método}

\section{Participantes}

Estudo de delineamento transversal foi realizado em 2011 (fevereiro a agosto). O universo amostral incluiu todos os bombeiros de Belo Horizonte, com pelo menos um ano de antiguidade na corporação. Foram considerados inelegíveis os bombeiros do sexo feminino $(n=70)$, em férias-prêmio ou em licença médica durante o período de coleta de dados $(n=40)$, os cedidos para outras unidades $(n=30)$ e aqueles que participaram da etapa piloto $(n=30)$. Os bombeiros do sexo feminino foram excluídos do estudo devido ao reduzido número no efetivo (7,3\% dos trabalhadores).

Buscou-se obter a adesão de pelo menos $80 \%$ para cada batalhão $(n=3)$ e subunidade $(n=11)$. Os participantes leram e assinaram o termo de consentimento livre e esclarecido após serem informados quanto aos objetivos e ao caráter confidencial e voluntário da pesquisa. O projeto foi aprovado pelo Corpo de Bombeiros de Minas Gerais e pelo Comitê de Ética em Pesquisa da Universidade Federal de Minas Gerais (ETIC no 0387.0.203.000-10).

\section{Instrumentos}

O desfecho depressão foi avaliado pelo Inventário Beck para Depressão (IBD) 18. O IBD é um instrumento de autorrelato para avaliação de sintomas de depressão, composto por 21 itens, 
com quatro opções de resposta. A elaboração do IBD incluiu critérios selecionados com base em observações e relatos de sintomas frequentes em pacientes psiquiátricos, incluindo aspectos cognitivos, emocionais e somáticos da doença. O escore total pode variar de 0 a 63 e permite a classificação da intensidade dos sintomas: (a) 0 a 11 - ausência de sintomas depressivos ou depressão mínima; (b) 12 a 19 - sintomas depressivos leves a moderados; (c) 20 a 35 - sintomas depressivos moderados a graves; e (d) 36 a 63 sintomas depressivos graves. $\mathrm{O}$ instrumento original foi desenvolvido para contextos clínicos, entretanto, estudos subsequentes indicaram que as características psicométricas do IBD são adequadas para abordar a população geral. Os estudos de tradução, validação e normatização da versão brasileira do IBD indicam características adequadas de validade e confiabilidade 19 . No presente estudo, a prevalência de depressão foi estimada pelo escore final no IBD (casos = escore total $>20$ ), pois é o escore mais adequado em amostras não clínicas 19. Recomendação semelhante já havia sido apresentada para a versão original do IBD 20 . Por essa razão, preferimos, para identificação de casos pelo IBD, optar por um ponto de corte mais específico do que sensível.

A Posttraumatic Diagnostic Scale, adaptada para profissionais de emergências (PDS-PE) 21, foi utilizada para avaliar a exposição a estressores operacionais. $\mathrm{O}$ instrumento consiste em uma lista de 15 estressores típicos dos serviços de emergências. $\mathrm{O}$ respondente indica a frequência com que vivenciou eventos durante o trabalho nos últimos 12 meses e qual deles mais o incomodou 22. A PDS-PE foi incluída nas análises como variável dicotômica. Alta exposição a estressores operacionais foi atribuída aos respondentes com escores acima do 4 o quintil na escala.

O Job Content Questionnaire (JCQ), adaptado para o português 23 , permitiu avaliar estressores organizacionais. Esse instrumento foi construído para operacionalizar o Modelo DemandaControle (Modelo DC) e inclui as dimensões demanda física e psicológica, controle sobre o trabalho e apoio social (http://www.jcqcenter. org). O modelo pressupõe a presença de quatro situações de trabalho relacionadas às dimensões demanda e controle: trabalho de baixa exigência, trabalho passivo, trabalho ativo e trabalho de alta exigência. Entretanto, o suporte empírico para o uso dos quadrantes em estudos epidemiológicos é limitado 24 . A estratégia de análise focada nos efeitos isolados de cada dimensão tem sido considerada mais promissora 25 Por essa razão, optou-se por analisar apenas os efeitos principais do Modelo DC.
As dimensões do JCQ foram incluídas nas análises como variáveis dicotômicas. $\mathrm{O}$ valor 1 foi atribuído para os participantes com escores pertencentes ao 5o quintil na dimensão demanda (alta demanda física/psicológica). Nas dimensões controle e apoio social, o valor 1 foi atribuído aos respondentes com escores pertencentes ao 1o quintil (baixo controle sobre o trabalho e apoio social escasso, respectivamente).

Condições do ambiente de trabalho foram investigadas por meio de itens referentes a diferentes fatores: disponibilidade de equipamentos de proteção individual, ruído no local de trabalho, ruído originado fora do local de trabalho e disponibilidade e adequação de recursos materiais para executar as tarefas. As respostas positivas aos itens mencionados foram somadas para a construção de um escore composto e analisadas como variável ordinal.

As seguintes características sociodemográficas foram estudadas: idade, estado civil, número de filhos, escolaridade, raça/cor da pele autodeclarada e renda mensal. A antiguidade na instituição e a posição hierárquica também foram abordadas.

A presença de sintomas de estresse pós-traumático foi realizada por meio da versão em português da Posttraumatic Stress Disorder Checklist (PCL) 26,27. A PCL é um questionário estruturado de autorrelato, composto por 17 itens congruentes com os sintomas de estresse pós-traumático 27. O respondente indica o quanto ele foi incomodado no último mês pelos sintomas descritos, utilizando uma escala likert de intensidade que varia de um (nada) a cinco (muito). O escore final pode variar de 17 a 85 pontos. O escore total maior ou igual a 50 pontos é recomendado como ponto de corte ótimo para presença de estresse pós-traumático 27.

Inquiriu-se sobre diagnóstico médico de ansiedade nos últimos 12 meses, diagnóstico de doenças crônicas (hipertensão, diabetes, asma/ bronquite, infarto do miocárdio, enfisema e distúrbios musculoesqueléticos), hábito de fumar e uso problemático de álcool, avaliado pelo questionário CAGE (Cut Down, Annoyed, Guilty, and Eye-opener) 28.

\section{Análise}

As análises foram realizadas no Stata, versão 11.0 (Stata Corp., College Station, Estados Unidos), em três etapas. Inicialmente, foi conduzida uma análise descritiva que incluiu o cálculo de porcentagens para variáveis ordinais e nominais. Em seguida, foi realizada uma análise de regressão logística univariável. As variáveis associadas ao IBD na análise univariável a um nível de signifi- 
cância igual ou menor que $20 \%(\mathrm{p}<0,20)$ foram incluídas na análise multivariável. Na última etapa, foi conduzida uma análise de regressão logística multivariável, com retirada manual de variáveis (procedimento de eliminação backward). Todas as variáveis associadas à depressão ao nível de significância igual ou menor a 5\% $(\mathrm{p}<0,05)$ foram mantidas no modelo final.

\section{Resultados}

Foram considerados elegíveis 794 bombeiros operacionais, em um universo de 954; a taxa de resposta foi $89,5 \%(n=711)$. Os resultados satisfizeram os critérios estabelecidos de quotas por batalhão e subunidade. A comparação entre respondentes e não respondentes indicou similaridades quanto a idade $(\mathrm{p}=0,106)$, escolaridade ( $\mathrm{p}$ $=309)$, estado civil $(\mathrm{p}=0,677)$, posto hierárquico ( $p=0,113)$, unidade de trabalho $(p=0,218)$ e tempo de serviço no Corpo de Bombeiros $(p=0,117)$.

\section{Análise descritiva}

Predominaram os participantes na faixa etária de 20-29 anos (40,2\%), com nível médio de escolaridade $(66 \%)$, casados ou em um relacionamento estável $(55,3 \%)$, com filhos $(52,5 \%)$, cor da pele parda $(51,6 \%)$ e renda familiar entre $\mathrm{R} \$ 2.501,00$ e R \$ 4.000,00 (41,8\%).

Observou-se predomínio de respondentes com 3 anos de antiguidade na instituição (64\%) e ocupando o posto de soldado (46\%). Em relação às características do trabalho, 50,2\% dos bombeiros relataram vivenciar duas ou mais condições precárias; 131 (19\%) sujeitos estavam expostos a alta demanda física e/ou psicológica; $144(21,1 \%)$, a baixo controle sobre as tarefas; 178 (25,8\%) contavam com fraco apoio social, e 155 $(23,2 \%)$ relataram alta exposição a estressores operacionais.

Quase um quinto dos sujeitos relatou diagnóstico clínico confirmado por médico de pelo menos uma doença crônica; 6,9\% apresentaram sintomas compatíveis com o diagnóstico de estresse pós-traumático; $8,1 \%$ indicaram problemas de ansiedade no passado; $9,2 \%$, uso problemático de álcool, e 7,6\% eram fumantes no momento do inquérito. A análise descritiva completa foi apresentada na Tabela 1.

\section{Prevalência e fatores associados}

A prevalência de depressão encontrada foi de 5,5\% (intervalo de 95\% de confiança - IC95\%: $4,1 \%-7,5 \%)$. Cento e vinte e oito bombeiros $(18,4 \%)$ apresentaram ao menos sintomas le- ves da doença, segundo seus relatos. Na análise univariável encontraram-se maiores taxas de depressão no grupo entre 25 e 39 anos de idade e entre aqueles com filhos. Bombeiros solteiros apresentaram uma taxa menor de adoecimento (Tabela 2).

Antiguidade na instituição, ocupar o posto de cabo, baixo controle sobre as tarefas, baixo apoio social e alta exposição a estressores operacionais foram positivamente associados à depressão na análise univariável. As seguintes morbidades foram associadas ao desfecho: estresse pós-traumático, diagnóstico de ansiedade no passado e diagnóstico de duas ou mais doenças crônicas. Quanto aos estilos de vida, tabagismo (passado e atual) e histórico de uso problemático de álcool foram positivamente associados à depressão (Tabela 2).

O modelo final multivariável incluiu variáveis relacionadas à situação de saúde. Bombeiros com sintomas compatíveis com diagnóstico de estresse pós-traumático (OR = 12,47; IC95\%: 5,64-27,57; valor de $\mathrm{p}<0,001)$ e histórico de uso problemático de álcool (OR = 5,30; IC95\%: 2,3511,96; valor de $\mathrm{p}<0,001$ ) apresentaram maior prevalência de depressão.

\section{Discussão}

O presente estudo investigou a prevalência e fatores associados à depressão em bombeiros de Belo Horizonte. Os resultados indicaram baixa prevalência (5,5\%) do desfecho na amostra estudada se comparada aos dados nacionais: $10,4 \%$ no inquérito populacional realizado em São Paulo 29. Como esperado, sintomas compatíveis com estresse pós-traumático e uso problemático de álcool foram associados à doença no modelo multivariável final.

Parâmetros distintos para a construção da variável desfecho, critérios de elegebilidade da amostra e a disparidade de instrumentos utilizados têm sido evocados para explicar discrepâncias entre os resultados obtidos nos estudos. Comparando com as características do inquérito de São Paulo, destacam-se algumas diferenças em relação ao nosso estudo: a prevalência na amostra de São Paulo refere-se ao período de 12 meses; diferentemente do grupo de bombeiros que é essencialmente masculino, ativo e empregado com vínculo formal, a amostra de São Paulo inclui mulheres, inativos ou desempregados. Os elementos metodológicos citados podem explicar parcialmente a variação nas taxas de adoecimento. Sabe-se que a depressão é mais frequente em mulheres do que em homens 1, e entre inativos ou desempregados quando comparados aos 
Tabela 1

Características sociodemográficas, estressores ocupacionais e situação de saúde de bombeiros de Belo Horizonte, Minas Gerais, Brasil.

\begin{tabular}{|c|c|c|}
\hline Variáveis & $\mathrm{n}$ & $\%$ \\
\hline \multicolumn{3}{|l|}{ Idade (anos) } \\
\hline $19-24$ & 161 & 22,7 \\
\hline $25-29$ & 124 & 17,5 \\
\hline $30-39$ & 226 & 31,9 \\
\hline $40-50$ & 198 & 27,9 \\
\hline \multicolumn{3}{|l|}{ Escolaridade } \\
\hline Ensino Fundamental & 54 & 7,6 \\
\hline Ensino Médio & 468 & 66,0 \\
\hline Ensino Superior/Pós-graduação & 187 & 26,4 \\
\hline \multicolumn{3}{|l|}{ Estado civil } \\
\hline Casado/União estável & 394 & 55,4 \\
\hline Solteiro & 284 & 39,9 \\
\hline Separado/Divorciado & 33 & 4,6 \\
\hline \multicolumn{3}{|l|}{ Filhos } \\
\hline Não & 333 & 46,9 \\
\hline Sim & 377 & 53,1 \\
\hline \multicolumn{3}{|l|}{ Raça/Cor da pele } \\
\hline Branca & 216 & 30,5 \\
\hline Parda & 367 & 51,8 \\
\hline Preta & 97 & 13,7 \\
\hline Asiática/Indígena & 28 & 4,0 \\
\hline \multicolumn{3}{|l|}{ Renda } \\
\hline$\leq \mathrm{R} \$ 2.500$ & 170 & 23,9 \\
\hline $\mathrm{R} \$ 2.501-\mathrm{R} \$ 4.000$ & 296 & 41,6 \\
\hline $\mathrm{R} \$ 4.001-\mathrm{R} \$ 5.500$ & 132 & 18,6 \\
\hline $\mathrm{R} \$ 5.501-\mathrm{R} \$ 7.000$ & 62 & 8,7 \\
\hline$>\mathrm{R} \$ 7.000$ & 51 & 7,2 \\
\hline \multicolumn{3}{|l|}{ Antiguidade (anos) } \\
\hline$<3$ & 253 & 36,4 \\
\hline $3-16$ & 225 & 32,3 \\
\hline $17-30$ & 218 & 31,3 \\
\hline \multicolumn{3}{|l|}{ Posto hierárquico } \\
\hline Soldado & 322 & 45,3 \\
\hline Cabo & 152 & 21,4 \\
\hline Sargento & 190 & 26,7 \\
\hline Oficial & 47 & 6,6 \\
\hline \multicolumn{3}{|l|}{ Demanda (JCQ) } \\
\hline Baixa & 560 & 79,1 \\
\hline Alta & 148 & 20,9 \\
\hline \multicolumn{3}{|l|}{ Controle (JCO) } \\
\hline Alto & 551 & 78,8 \\
\hline Baixo & 148 & 21,2 \\
\hline \multicolumn{3}{|l|}{ Apoio social (JCQ) } \\
\hline Alto & 583 & 82,6 \\
\hline Baixo & 123 & 17,4 \\
\hline \multicolumn{3}{|l|}{ Eventos traumáticos (PDS) } \\
\hline Baixa & 552 & 80,5 \\
\hline Alta & 134 & 19,5 \\
\hline
\end{tabular}

(continua) 
Tabela 1 (continuação)

\begin{tabular}{|c|c|c|}
\hline Variáveis & $\mathrm{n}$ & $\%$ \\
\hline \multicolumn{3}{|c|}{ Ambiente de trabalho (número de } \\
\hline \multicolumn{3}{|c|}{ condições precárias) } \\
\hline 0 & 81 & 11,5 \\
\hline 1 & 269 & 38,1 \\
\hline 2 ou mais & 356 & 50,4 \\
\hline \multicolumn{3}{|l|}{ Depressão (IBD) } \\
\hline Não & 653 & 94,5 \\
\hline Sim & 38 & 5,5 \\
\hline \multicolumn{3}{|c|}{ Uso abusivo de álcool (CAGE) } \\
\hline Não & 640 & 90,4 \\
\hline $\operatorname{Sim}$ & 68 & 9,6 \\
\hline \multicolumn{3}{|l|}{ Tabagismo } \\
\hline Não fumante & 587 & 82,6 \\
\hline Ex-fumante & 70 & 9,8 \\
\hline Fumante & 54 & 7,6 \\
\hline \multicolumn{3}{|c|}{ Estresse pós-traumático (PCL) } \\
\hline Não & 644 & 93,1 \\
\hline Sim & 48 & 6,9 \\
\hline \multicolumn{3}{|c|}{ Diagnóstico de ansiedade } \\
\hline Não & 647 & 91,6 \\
\hline $\operatorname{Sim}$ & 59 & 8,4 \\
\hline \multicolumn{3}{|l|}{ Doenças crônicas } \\
\hline 0 & 555 & 79,3 \\
\hline 1 & 115 & 16,4 \\
\hline 2 ou mais & 30 & 4,3 \\
\hline
\end{tabular}

CAGE: Cut Down, Annoyed, Guilty and Eye-opened; IBD: Inventário de Beck para Depressão; JCQ: Job Content

Questionnaire; PCL: Posttraumatic Stress Disorder Checklist; PDS: Posttraumatic Diagnostic Scale.

trabalhadores ativos 7. Trabalhadores com instabilidade no emprego e sem proteção social apresentam piores resultados em saúde mental, incluindo sintomas psiquiátricos inespecíficos 30 , ansiedade ${ }^{8}$ e depressão ${ }^{7}$. Por fim, não há concordância quanto ao instrumento utilizado. Em São Paulo, a depressão foi detectada por meio do Composite International Diagnostic Interview (WMH-CIDI), um instrumento diagnóstico mais robusto que o IBD.

A prevalência no grupo dos bombeiros de Belo Horizonte foi menor do que a encontrada em outros estudos. Em Juiz de Fora 17, Japão $13 \mathrm{e}$ Estados Unidos 31 registraram-se: 8,3\%, 22,3\% e $27 \%$, respectivamente. Se ampliarmos o espectro da comparação para outras profissões do setor de emergências, as prevalências são maiores em trabalhadores de ambulância tanto no Canadá 12 quanto na Inglaterra 32: $24 \%$ e $9 \%$, respectivamente. Em médicos de unidades de emergências da Turquia, a prevalência também foi maior $(15,1 \%) 33$. Esses estudos se assemelham quanto ao delineamento (transversal) e ao uso de ins- trumentos de autorrelato para mensuração de sintomas da doença. Não obstante, as diferenças podem ter sido influenciadas pelas divergências quanto aos critérios para definição de casos 15 . Tal limitação é evidente no estudo conduzido em Juiz de Fora, cujo ponto de corte no IBD foi menos estrito 17 .

Vale contudo ressaltar que as hipóteses aventadas são incipientes, haja vista, por exemplo, que a diferença de prevalência ( $9 \%$ versus $5,5 \%$ em Belo Horizonte) também foi atestada no estudo que focalizou enfermeiros no Estado de São Paulo 34 , ainda que tenham sido utilizados critérios idênticos aos nossos.

Estaria presente o efeito do trabalhador sadio 35? No serviço operacional do Corpo de Bombeiros Militar de Minas Gerais são rígidos os critérios de seleção de saúde para entrada e permanência. Os bombeiros são submetidos anualmente a provas de resistência e força física, cujo desempenho influencia tanto a ascensão na carreira quanto a remuneração (Corpo de Bombeiros Militar de Minas Gerais. Resolução 
Tabela 2

Prevalência e fatores associados à depressão em bombeiros de Belo Horizonte, Minas Gerais, Brasil (análise univariável).

\begin{tabular}{|c|c|c|c|}
\hline Variáveis & $\begin{array}{l}\text { Bombeiros com depressão } \\
n(\%)\end{array}$ & OR & IC95\% \\
\hline \multicolumn{4}{|l|}{ Idade (anos) } \\
\hline $19-24$ & $5(3,1)$ & 1,00 & - \\
\hline $25-29$ & $9(7,3)$ & 2,45 & $0,80-7,50$ * \\
\hline $30-39$ & $14(6,4)$ & 2,13 & $0,75-6,03$ * \\
\hline $40-50$ & $10(5,3)$ & 1,74 & $0,58-5,21$ \\
\hline \multicolumn{4}{|l|}{ Escolaridade } \\
\hline Ensino Fundamental & $3(6,3)$ & 1,00 & - \\
\hline Ensino Médio & $24(5,2)$ & 0,83 & $0,24-2,86$ \\
\hline Ensino Superior/Pós-graduação & $11(6,0)$ & 0,96 & $0,26-3,58$ \\
\hline \multicolumn{4}{|l|}{ Estado civil } \\
\hline Casado/União estável & $26(6,8)$ & 1,00 & - \\
\hline Solteiro & $11(4,0)$ & 0,57 & $0,27-1,17$ * \\
\hline Separado/Divorciado & $1(3,1)$ & 0,44 & $0,06-3,37$ \\
\hline \multicolumn{4}{|l|}{ Filhos } \\
\hline Não & $10(3,1)$ & 1,00 & - \\
\hline Sim & $28(7,7)$ & 2,67 & $1,27-5,58$ ** \\
\hline \multicolumn{4}{|l|}{ Raça/Cor da pele } \\
\hline Branca & $13(6,1)$ & 1,00 & - \\
\hline Parda & $18(5,1)$ & 0,83 & $0,40-1,72$ \\
\hline Preta & $6(6,6)$ & 1,09 & $0,40-2,97$ \\
\hline Asiática/Indígena & $1(3,6)$ & 0,57 & $0,07-4,55$ \\
\hline \multicolumn{4}{|l|}{ Renda } \\
\hline$\leq \mathrm{R} \$ 2.500$ & $9(5,5)$ & 1,00 & - \\
\hline $\mathrm{R} \$ 2.501-\mathrm{R} \$ 4.000$ & $17(5,9)$ & 1,08 & $0,47-2,49$ \\
\hline $\mathrm{R} \$ 4.001-\mathrm{R} \$ 5.500$ & $7(5,5)$ & 1,00 & $0,36-2,77$ \\
\hline $\mathrm{R} \$ 5.501-\mathrm{R} \$ 7.000$ & $2(3,4)$ & 0,61 & $0,13-2,90$ \\
\hline$>\mathrm{R} \$ 7.000$ & $3(6,0)$ & 1,11 & $0,29-4,25$ \\
\hline \multicolumn{4}{|l|}{ Antiguidade (anos) } \\
\hline$<3$ & $8(3,2)$ & 1,00 & - \\
\hline $3-16$ & $16(7,3)$ & 2,41 & $1,00-5,74^{\star \star}$ \\
\hline $17-30$ & $14(6,7 \%$ & 2,19 & $0,90-5,33^{*}$ \\
\hline \multicolumn{4}{|l|}{ Posto hierárquico } \\
\hline Soldado & $13(4,1)$ & 1,00 & - \\
\hline Cabo & $13(9,0)$ & 2,33 & $1,05-5,16^{\star *}$ \\
\hline Sargento & $9(5,0)$ & 1,22 & $0,51-2,91$ \\
\hline Oficial & $3(6,4)$ & 1,60 & $0,44-5,84$ \\
\hline \multicolumn{4}{|l|}{ Demanda (JCQ) } \\
\hline Baixa & $27(5,0)$ & 1,00 & - \\
\hline Alta & $11(7,6)$ & 1,59 & $0,77-3,29$ \\
\hline \multicolumn{4}{|l|}{ Controle (JCQ) } \\
\hline Alto & $25(4,7)$ & 1,00 & - \\
\hline Baixo & $13(9,0)$ & 2,04 & $1,01-4,09^{* *}$ \\
\hline \multicolumn{4}{|l|}{ Suporte social (JCQ) } \\
\hline Alto & $25(4,4)$ & 1,00 & - \\
\hline Baixo & $13(11,0)$ & 2,70 & $1,34-5,46$ ** \\
\hline \multicolumn{4}{|l|}{ Eventos traumáticos (PDS) } \\
\hline Baixa & $24(4,4)$ & 1,00 & - \\
\hline Alta & $13(10,2)$ & 2,44 & $1,20-4,93 * \star$ \\
\hline
\end{tabular}




\begin{tabular}{|c|c|c|c|}
\hline Variáveis & $\begin{array}{c}\text { Bombeiros com depressão } \\
\qquad \mathrm{n}(\%)\end{array}$ & OR & IC95\% \\
\hline \multicolumn{4}{|c|}{$\begin{array}{l}\text { Ambiente de trabalho (número de condições } \\
\text { precárias) }\end{array}$} \\
\hline 0 & $3(3,8)$ & 1,00 & - \\
\hline 1 & $12(4,6)$ & 1,23 & $0,34-4,48$ \\
\hline 2 ou mais & $23(6,7)$ & 1,84 & $0,54-6,28$ \\
\hline \multicolumn{4}{|c|}{ Uso abusivo de álcool } \\
\hline Não & $24(3,8)$ & 1,00 & - \\
\hline Sim & $14(22,2)$ & 7,15 & $3,48-14,71$ ** \\
\hline \multicolumn{4}{|l|}{ Tabagismo } \\
\hline Não fumante & $24(4,2)$ & 1,00 & - \\
\hline Ex-fumante & $7(10,5)$ & 2,66 & $1,10-6,44$ ** \\
\hline Fumante & $7(13,5)$ & 3,55 & $1,45-8,69$ ** \\
\hline \multicolumn{4}{|c|}{ Estresse pós-traumático (PCL) } \\
\hline Não & $20(3,2)$ & 1,00 & - \\
\hline Sim & $16(34,8)$ & 16,21 & $7,63-34,41$ ** \\
\hline \multicolumn{4}{|c|}{ Diagnóstico de ansiedade } \\
\hline Não & $27(4,3)$ & 1,00 & - \\
\hline Sim & $11(19,6)$ & 5,48 & $2,55-11,76$ ** \\
\hline \multicolumn{4}{|l|}{ Doenças crônicas } \\
\hline 0 & $24(4,4)$ & 1,00 & - \\
\hline 1 & $8(7,2)$ & 1,68 & $0,73-3,84$ \\
\hline 2 ou mais & $6(21,4)$ & 5,90 & $2,19-15,89$ ** \\
\hline
\end{tabular}

Variável dependente: depressão (IBD - escore total $\geq 20$ );

* Variáveis associadas à depressão na análise univariável ( $p \leq 0,20)$;

** Variáveis associadas à depressão na análise univariável $(p \leq 0,05)$

CAGE: Cut Down, Annoyed, Guilty and Eye-opened; IBD: Inventário de Beck para Depressão; IC95\%: intervalo de 95\% de confiança; JCQ: Job Content Questionnaire; OR: odds ratio; PCL: Posttraumatic Stress Disorder Checklist; PDS: Posttraumatic Diagnostic Scale.

no 114 de 31 de dezembro de 2003, que dispõe sobre o Teste de Avaliação Física para ingresso no Corpo de Bombeiros Militar de Minas Gerais). Tal realidade parece não marcar a trajetória dos profissionais de emergências em outros setores (hospitais, por exemplo).

A lógica subjacente ao efeito do trabalhador sadio traduz a lógica da demanda e oferta do mercado de trabalho, ou seja, pessoas inicialmente saudáveis são mais ativas na busca de um emprego (autosseleção) e mais facilmente selecionadas (seleção pelo empregador). Contudo, quando adoecem, enfrentam margens estreitas para manter o cargo ou a função, sendo comuns: licença-saúde, transferência para o setor administrativo ou aposentadoria precoce 36 .

Hábitos de vida saudáveis possivelmente exacerbam as diferenças entre grupos ocupacionais específicos 36 . É provável que os bombeiros adiram mais facilmente às práticas saudáveis fora do trabalho como atividade física e alimenta- ção equilibrada (dados não publicados). Estilos de vida saudáveis podem estar associados a estratégias de enfrentamento que são cruciais em situações ocupacionais estressantes, como é o caso dos bombeiros. Em suma, as exigências do trabalho, os mecanismos de funcionamento do grupo e as regras da instituição podem selecionar, de forma contínua, os bombeiros mais saudáveis e resilientes 35 .

O reconhecimento social da atividade dos bombeiros seria ainda um potente fator de proteção contra o sofrimento no trabalho, o qual teria relação com a saúde 37 . A retribuição diante do engajamento na atividade é essencialmente simbólica e pode ser proferida por chefes, colegas, subordinados ou usuários dos serviços. O reconhecimento é atrelado a julgamentos sobre a qualidade do serviço prestado com efeitos sobre o indivíduo, sua identidade e bem-estar. Vale ressaltar a confiança e apreço das populações aos bombeiros que, em pesquisas de opinião pública 
no país (http://www.ibope.com.br), são identificados como "heróis" e "amigos" (http://www. cbmmg.mg.gov.br). Seria plausível pensar que diante do reconhecimento social, os bombeiros estariam mais fortalecidos para enfrentar as vicissitudes e traumas que caracterizam sua atividade, estando assim menos vulneráveis ao adoecimento? Os nossos resultados sugerem que sim.

Quanto aos fatores associados, o estresse póstraumático aumentou a chance de depressão no modelo final (OR = 12,47; IC95\%: 5,64-27,57). Dezesseis (33,3\%) entre os 48 participantes com estresse pós-traumático também apresentaram sintomas moderados ou graves de depressão. Adicionalmente, a exposição a eventos traumáticos ocupacionais aumentou a chance de estresse pós-traumático na amostra estudada 22 . Tal associação não foi observada entre eventos traumáticos e depressão.

Comparado à depressão, o estresse pós-traumático está mais diretamente ligado a eventos traumáticos. Pelo menos três modelos explicativos são considerados nas relações entre os dois desfechos 16 . O primeiro hipotetiza que a exposição a estressores operacionais explicaria ambas as doenças de forma independente; o segundo afirma que o diagnóstico prévio de depressão aumentaria a suscetibilidade dos indivíduos para o desenvolvimento de sintomas de estresse póstraumático; o terceiro indica que a depressão seria uma complicação decorrente do estresse póstraumático. Nossos resultados vão ao encontro deste último modelo.

O uso de álcool e de outras substâncias psicoativas pode aumentar o risco de transtornos mentais (hipótese fator de risco). Nesses casos, não está descartado que o uso de substâncias seja uma estratégia comportamental para lidar com eventos traumáticos (hipótese automedicação) ${ }^{38}$. Essa última explicação pode ser útil para entender os nossos resultados. Bombeiros com histórico de uso problemático de álcool tiveram uma chance quase cinco vezes maior $(\mathrm{OR}=4,59$; IC95\%: 1,82-11,57) de apresentar sintomas moderados ou graves de depressão.

As associações diretas e significativas entre estresse pós-traumático, uso problemático de álcool e depressão no modelo multivariado final reforçam a hipótese quanto ao efeito do trabalhador sadio. Se estresse pós-traumático é um fator de risco para depressão e se o uso de substâncias funciona como paliativo para os efeitos do estresse, é possível que os sujeitos em tais condições não tenham mantido os seus postos de trabalho. Aqueles com alterações comportamentais e, consequentemente, com alto risco pa- ra depressão teriam sido licenciados ou transferidos para setores administrativos da instituição. Contudo, não foi possível examinar tal hipótese.

A ausência de associações entre estressores ocupacionais e depressão não é convergente com a literatura 32,39. Lembrar que baixo controle sobre o trabalho, fraco apoio social e alta exposição a eventos traumáticos foram positivamente associados ao desfecho nas análises univariáveis, mas não permaneceram no modelo final. Seria efeito do desenho transversal?

A antiguidade é um fator relevante na eclosão dos sintomas psíquicos. No caso, constatou-se baixa antiguidade dos respondentes (3 anos para $40 \%$ da amostra). Ora, o surgimento de sintomas de depressão e de outros transtornos mentais depende da duração da exposição a estressores organizacionais 40 . Sintomas inespecíficos e estratégias disfuncionais para enfrentar adversidades no ambiente de trabalho (tabagismo, uso abusivo de álcool, entre outros) poderiam funcionar como mediadores de maneira a retardar os quadros de depressão 16,38.

As considerações sobre efeito do trabalhador sadio e tempo de exposição ocupacional são limitadas diante do caráter transversal do estudo. Uma segunda limitação refere-se ao instrumento de mensuração de sintomas (IBD). O ponto de corte adotado levou à inclusão de casos leves de depressão entre os indivíduos não doentes. Tal escolha pode ter comprometido o poder do estudo de identificar associações. Entretanto, o IDB é um instrumento de rastreio, não tendo sido construído para diagnosticar depressão 19. Por isso, recomenda-se um ponto de corte mais restritivo quando se busca rastrear depressão 19,20. Para contornar essa barreira, adotou-se um ponto de corte conservador visando evitar a inclusão de falsos positivos

\section{Conclusão}

O inquérito de saúde identificou uma baixa prevalência de depressão (5,5\%). O modelo logístico multivariável indicou associações fortes e estatisticamente significantes entre estresse pós-traumático, uso problemático de álcool e presença de depressão. Os resultados permitiram discutir as inter-relações entre transtornos mentais, reconhecimento profissional e seleção de saúde em bombeiros. A hipótese de um viés na estimativa de prevalência (efeito do trabalhador sadio) foi aventada. Estudos prospectivos são necessários para compreender essas associações e os mecanismos nelas implicados. 


\section{Resumen:}

La depresión tiene una alta carga como enfermedad mundial. Factores socioeconómicos y la exposición a situaciones extremas en el trabajo pueden estar asociados con la enfermedad. El objetivo de este trabajo es investigar la prevalencia y los factores asociados con la depresión en los bomberos de Belo Horizonte, Minas Gerais, Brasil. Se trata de un estudio transversal, realizado entre los bomberos de sexo masculino de Belo Horizonte $(n=711)$. Se utilizó el Inventario de Depresión de Beck (IDB) para evaluar la presencia de depresión. Se utilizaron modelos de regresión logística para estudiar la asociación entre características sociodemográficas, estrés ocupacional, estado de salud y depresión. La prevalencia de depresión fue de un 5,5\%. La posibilidad de depresión fue mayor entre los bomberos que informaron síntomas de estrés postraumático $(\mathrm{OR}=$ 12,47; IC95\%: 5,64-27,57) y abuso de alcohol $(O R=5,30$, IC95\%: 2,35-11,96). Los resultados son discutidos considerando las interrelaciones entre los trastornos mentales, el efecto en trabajadores sanos y el reconocimiento social de la labor de bomberos.

Bomberos; Exposición Profesional; Depresión; Efecto del Trabajador Sano

\section{Colaboradores}

E. P. Lima, A. A. Assunção e S. M. Barreto contribuíram na concepção e desenho do estudo; análise e interpretação dos dados; redação e revisão do texto; aprovação da versão final.

\section{Agradecimentos}

Agradecemos ao CNPq pela bolsa sanduíche concedida à Eduardo de Paula Lima durante o doutorado e ao Corpo de Bombeiros Militar de Minas Gerais pelo apoio na realização da pesquisa.

\section{Referências}

1. Bromet E, Andrade LH, Hwang I, Sampson NA, Alonso J, de Girolamo G, et al. Cross-national epidemiology of DSM-IV major depressive episode. BMC Med 2011; 9:90.

2. American Psychiatric Association. Diagnostic and statistical manual of mental disorders. $5^{\text {th }} \mathrm{Ed}$ Washington DC: American Psychiatric Association; 2013.

3. Salguero JM, Fernández-Berrocal P, Iruarrizaga I Cano-Vindel A, Galea S. Major depressive disorder following terrorist attacks: a systematic review of prevalence, course and correlates. BMC Psychiatry 2011; 11:96.

4. Nestler EJ, Barrot M, DiLeone RJ, Eisch AJ, Gold SJ, Monteggia LM. Neurobiology of depression. Neuron 2002; 34:13-25.

5. Murray CJ, Vos T, Lozano R, Naghavi M, Flaxman AD, Michaud C, et al. Disability-adjusted life years (DALYs) for 291 diseases and injuries in 21 regions, 1990-2010: a systematic analysis for the Global Burden of Disease Study 2010. Lancet 2012; 380:2197-223.

6. World Health Organization. The global burden of disease: 2004 update. Geneva: World Health Organization; 2008.

7. Kim SS, Subrumanian SV, Sorensen G, Perry MJ, Christiani DC. Association between change in employment status and new-onset depressive symptoms in South Korea - a gender analysis. Scand J Work Environ Health 2012; 38:537-45.

8. Strazdins L, D'Souza RM, Clements M, Broom DH, Rodgers B, Berry HL. Could better jobs improve mental health? A prospective study of change in work conditions and mental health in mid-aged adults. J Epidemiol Community Health 2011;65:529-34.

9. Grynderup MB, Mors O, Hansen AM, Andersen JH, Bonde JP, Kærgaard A, et al. A two-year follow-up study of risk of depression acoording to work-unit measures of psychological demands and decision latitude. Scand J Work Environ Health 2012; 38:527-36.

10. Netterson B, Conrad N, Bech P, Fink P, Olsen O, Rugulies $\mathrm{R}$, et al. The relation between work-related psychosocial factors and the development of depression. Epidemiol Rev 2008;30:118-32.

11. Strathopoulou H, Karanikola MNK, Panagiotopoulou F, Papathianassoglou EDE. Anxiety levels and related symptoms in emergency nursing personnel in Greece. J Emerg Nurs 2011; 37:314-20.

12. Halpern J, Maunder RG, Schwartz B, Gurevich M. Identifying risk of emotional sequelae after critical incidents. Emerg Med J 2011; 28:51-6.

13. Saijo Y, Ueno T, Hashimoto Y. Twenty-four-hour shift work, depressive symptoms, and job dissatisfaction among Japanese firefighters. Am J Ind Med 2008; 51:380-91.

14. de Boer J, Lok A, Van't Verlaat E, Duivenvoorden HJ, Bakker AB, Smit BJ. Work-related critical incidents in hospital-based health care providers and the risk of post-traumatic stress symptoms, anxiety, and depression: a meta-analysis. Soc Sci Med 2011; 73:316-26. 
15. Berger W, Coutinho ESF, Figueira I, Marques-Portella C, Luz MP, Neylan TC, et al. Rescuers at risk: a systematic review and meta-regression analysis of the worldwide current prevalence and correlates of PTSD in rescue workers. Soc Psychiatry Psychiatr Epidemiol 2012; 47:1001-11.

16. Ginzburg K, Ein-Dor T, Solomon Z. Comorbidity of posttraumatic stress disorder, anxiety and depression: a 20-year longitudinal study of war veterans. J Affect Disord 2010; 123:249-57.

17) Amato TC, Pavin T, Martins LF, Batista A, Ronzani TM. Trabalho, gênero e saúde mental: uma pesquisa quantitativa e qualitativa entre bombeiros. Cad Psicol Soc Trab 2010; 13:103-18.

18. Beck AT, Ward CH, Mendelson M, Mock J, Erbaugh J. An inventory for measuring depression. Arch Gen Psychiatry 1961; 4:561-71.

19. Cunha JA. Manual da versão em português das Escalas Beck. São Paulo: Casa do Psicólogo; 2001.

20. Kendall PC, Hollon SD, Beck AT, Hammen CL, Ingram RE. Issues and recommendations regarding use of the Beck Depression Inventory. Cognit Ther Res 1987; 11:289-99.

21. Laposa JM, Alden LE. Posttraumatic stress disorder in the emergency room: exploration of a cognitive model. Behav Res Ther 2003; 41:49-65.

22. Lima EP. Transtorno de estresse pós-traumático (TEPT) em bombeiros de Belo Horizonte [Tese de Doutorado]. Belo Horizonte: Universidade Federal de Minas Gerais; 2013

23. Araújo TM, Karasek R. Validity and reliability of the job content questionnaire in formal and informal jobs in Brazil. Scand J Work Env Hea Suppl 2008; 6:52-9.

24. de Lange AH, Taris TW, Kompier MA, Houtman IL, Bongers PM. "The very best of the millennium": longitudinal research and the demand-control(support) model. J Occup Health Psychol 2003; 8:282-305.

25. De Jonge J, Kompier MAJ. A critical examination of the demand-control-support model from a work psychological perspective. Int J Stress Manag 1999; 4:235-58.

26. Berger W, Mendlowicz MV, Souza WF, Figueira I. Equivalência semântica da versão em português da Post-Traumatic Stress Disorder Checklist - Civilian Version (PCL-C) para rastreamento do transtorno de estresse pós-traumático. Rev Psiquiatr Rio Gd Sul 2004; 26:167-75.

27. Lima EP, Barreto SM, Assunção AA. Factor structure, internal consistency and reliability of the Posttraumatic Stress Disorder Checklist (PCL): an exploratory study. Trends Pychiatry psychother 2012; 34:215-22.

28. Masur J, Monteiro MG. Validation of the "CAGE" alcoholism screening test in a Brazilian psychiatric inpatient hospital setting. Braz J Med Biol Res 1983; 16:215-8.
29. Kessler RC, Birnbaum HG, Shahly V, Bromet E, Hwang I, McLaughlin KA, et al. Age differences in the prevalence and co-morbidity of DSM-IV major depressive episodes: results from the WHO World Mental Health Survey Initiative. Depress Anxiety 2010; 27:351-64.

30. Dilélio AS, Facchini LA, Tomasi E, Silva SM, Thumé E, Piccini RX, et al. Prevalência de transtornos psiquiátricos menores em trabalhadores da atenção primária à saúde das regiões Sul e Nordeste do Brasil. Cad Saúde Pública 2012; 28:503-14.

31. Tak S, Driscoll R, Bernard B, West C. Depressive Symptoms among firefighters and related factors after the response to hurricane Katrina. J Urban Health 2007; 84:153-61.

32. Bennett P, Williams Y, Page N, Hood K, Woollard M, Vetter N. Associations between organizational and incident factors and emotional distress in emergency ambulance personnel. Br J Clin Psychol 2005; 44:215-26.

33. Erdur B, Ergin A, Turkcuer I, Parlak I, Ergin N, Boz B. A study of depression and anxiety among doctors working in emergency units in Denizili, Turkey. Emerg Med J 2006; 23:759-63.

34. Vargas D, Dias APV. Prevalência de depressão em trabalhadores de enfermagem de Unidade de Terapia Intensiva: estudo em hospitais de uma cidade do noroeste do Estado de São Paulo. Rev Latinoam Enferm 2011; 19:1114-21.

35. Shah D. Healthy worker effect phenomenon. Indian J Occup Environ Med 2009;13:77-9.

36. Koskela RS. Mortality, morbidity and health selection among mental workers. Scand J Work Environ Health 1997; 23 Suppl 2:1-80.

37. Dejours C. Addendum 1993. In: Lancman S, Sznelwar LI, editors. Christophe Dejours - da psicopatologia à psicodinâmica do trabalho. Rio de Janeiro: Editora Fiocruz/Brasilia: Paralelo 15; 2004. p. $47-104$

38. Bacharach SB, Bamberger PA, Doveh E. Firefighters, critical incidents, and drinking to cope: the adequacy of unit-level performance resources as a source of vulnerability and protection. J Appl Psychol 2008; 93:155-69.

39. Chen YS, Chen MC, Chou FHC, Sun FC, Chen PC, Tsai KY, et al. The relationship between quality of life and posttraumatic stress disorder or major depression for firefighters in Kaohsiung, Taiwan. Qual Life Res 2007; 16:1289-97.

40. Schnall PL, Dobson M, Rosskam E, editors. Unhealthy work: causes, consequences, cures. New York: Baywood Publishing Company; 2009.

Recebido em 02/Abr/2014

Versão final reapresentada em 14/Out/2014

Aprovado em 17/Nov/2014 
Lima EP, Assunção AA, Barreto SM. Prevalência de depressão em bombeiros. Cad Saúde Pública 2015; 31(4):733-743.

A revista foi informada sobre um erro no quarto parágrafo da Introdução (p. 734). O texto correto é: The journal has been informed of an error in the 4th paragraph of the Introduction (p. 734). The correct text is:

La revista fue informada sobre un error em el cuarto parágrafo de la Introducción (p. 734). El texto correcto es:

Bombeiros desempenham tarefas diversas em contextos de emergência. Combate a incêndio, atendimento pré-hospitalar e resgate de feridos em acidentes automobilísticos são exemplos de ocorrências rotineiras atendidas em centros urbanos. Tais demandas exigem respostas rápidas para assegurar a integridade física das vítimas e dos próprios trabalhadores, implicando convívio com situações extremas como morte iminente ou atendimento às zonas onde houve massacres $\mathrm{e}$ acidentes fatais 14 . 